\title{
CORRELATION BETWEEN FNAC AND OPEN BIOPSY IN CERVICAL LYMPHADENOPATHY IN CHILDREN
}

\author{
T HOSSAIN ${ }^{1}$, MR AMIN $^{2}, M_{T H}$ SIDDIQUI ${ }^{3}$, MAU HUQ $^{4}$, MM RAHMAN $^{5}$
}

\begin{abstract}
:
Background: Head and Neck region of the body has a rich network of lymphatic channel and intervening nodes. Out of total approximately 800 lymph nodes in the body about $\mathbf{3 0 0}$ are scattered in organized fashion in the neck region which are affected by various regional and systemic diseases. ${ }^{1}$

The conventional method of excisional biopsy is used to diagnose etiological causes of lymphadenopathy. In comparison to open surgical biopsy, FNAC is a simple, reliable and acceptable tool for the etiological diagnosis of lymphadenopathies.

FNAC was first practiced by Grieg and Gray for the diagnosis of trypanosome in lymphnode in 1904. Now a day it is widely practiced in United States, United Kingdom and many other countries. In Bangladesh, many studies on FNAC are carried out and the results are highly appreciable and reliable.

Methods: A prospective study for a period of 24 months was carried out on $\mathbf{5 0}$ patients with enlarged cervical lymph nodes from July 2003 to June 2005. During the two years study period, 56 patients with cervical lymphadenopathies were selected for FNAC and open
\end{abstract}

1. Dr. Tahmina Hossain, Asstt. Professor, Dept. of Pediatric Surgery, Dhaka Medical College \& Hospital

2. Dr. Md. Ruhul Amin, Associate Professor, Dept. of Pediatric Surgery, Bangabandhu Sheikh Mujib Medical University, Dhaka

3. Dr. MTH Siddiqui, Associate Professor, Dept. of Pediatric Surgery, Bangabandhu Sheikh Mujib Medical University, Dhaka

4. Dr. Md. Ashraf ul Huq, Associate Professor, Dept. of Pediatric Surgery, Dhaka Medical College Hospital, Dhaka

5. Dr. Md. Matiur Rahman, Professor, Dept. of Pediatric Surgery, Bangabandhu Sheikh Mujib Medical University, Dhaka

Correspondence to: Dr. Tahmina Hossain, Asstt. Professor, Dept. of Pediatric Surgery, Dhaka Medical College \& Hospital. E-mail: tahminahrahman@yahoo.com biopsy following certain inclusion and exclusion criteria, in the Department of Pediatric Surgery, Bangabandhu Sheikh Mujiib Medical University, Dhaka.

Patients with acute lymphadenitis and lymphadenopathy due to leukaemia were excluded.

Six cases were deleted from the study as smears from those revealed inadequate material and hence only $\mathbf{5 0}$ cases were available for study in the present series.

Clinical findings, FNAC and biopsy reports were available in all the cases and were correlated with each other.

Result: On biopsy, 27 cases were diagnosed as tuberculous lymphadenopathies, 15 cases as lymphoma and 8 cases as reactive changes.

On FNAC, there were 2 false positive and 5 false negative cases in case of tuberculosis; 2 false positive and 1 false negative cases in lymphoma; 4 false positive and 2 false negative cases in cases with reactive hyperplasia.

Sensitivity of FNAC in comparison to histopathological findings was $92.6 \%$ in case of tuberculosis; $86.7 \%$ in case of lymphoma and $50 \%$ in case of nonspecific lymphadenitis.

Specificity of FNAC in comparison to histological findings was $78.3 \%$ in case of tuberculosis; $94.2 \%$ in case of lymphoma and $\mathbf{9 5 . 3 \%}$ in case of nonspecific lymphadenitis. Accuracy of FNAC in comparison to histopathological findings was $86 \%$ in case of tuberculosis; $94 \%$ in case of lymphoma and $88 \%$ in case of reactive changes.

Conclusion: The present study indicates that, FNAC is a simple, reliable and acceptable procedure for various lesions of cervical lymph nodes. It can be repeated if necessary. It also concludes that FNAC helps to confirm the clinical impression without open biopsy. Open biopsy can be avoided in many benign and malignant lesions of cervical lymph nodes. Fine needle aspiration cytodiagnosis can eliminate the need of hospitalization and surgery. ${ }^{2}$

Keywords : FNAC, Cervical Lymphadenopathy. 


\section{Introduction:}

The lymphnodes represent the primary site of interaction between foreign and immunocompetent cells. ${ }^{3}$ Therefore, structural changes in the lymphnode morphology directly reflects local immune reactivity.

Lymphnodes less than $3 \mathrm{~mm}$ in diameter are defined as normal cervical ${ }^{4}$. During childhood there is rapid growth of lymphoid tissue and by early adolescence, achieve twice its adult size. But start regressing during mid adolescence and reaches the stable adult size by $20-25$ years of age. Therefore, palpable lymphnodes are commonly found in late childhood.

Enlarged lymphnodes are primary or secondary manifestations of numerous disorders varying from a local inflammatory process to systemic disease. Substantial enlargement of the lymphnode requiring investigation is defined as one or more lymphnodes estimated to be equal to or greater than $1 \mathrm{~cm}$ in diameter, newly recognized and not known to arise from previously recognized cause. ${ }^{1}$

The conventional method of excision biopsy is used to diagnose etiological causes of lymphadenopathy. This is an invasive procedure that requires some investigations, anesthesia and surgical procedure. The subsequent healing results in a scar formation. In a third world country, where health care facilities are grossly inadequate to meet the demand; rapid, easy, less expensive procedures are required to offer satisfactory answer for etiological diagnosis of lymphadenopathies.

Fine needle aspiration cytology (FNAC) is a simple technique, easy to perform, reliable method and can be performed in the outpatient department. The procedure is minimally invasive. It requires no anesthesia and in most cases, causes no complication not even bruising or bleeding. It needs no special preparation. Patients can go about the day after an FNAC as if they hadn't had the test. ${ }^{5}$

In the millennium, as health care dollars become less and less available, both patients and providers are aware of the need for cost-effective medicine. FNAC is perhaps the most cost-effective method for establishing a tissue diagnosis. ${ }^{6}$

Patients are informed consumers and it is very appealing to them to know that they can have a quick out-patient FNAC procedure, experience minimal discomfort, avoid an anesthetic, and in most cases can have a result within a few hours or in the same day. Traditional biopsies need to be processed before they can be sectioned for microscopic examination. This process requires a minimum of 24 hours before diagnosis. $^{7}$

So, FNAC with all its efficiency and advantages can be readily available to all the doctors and their patients in our country.

\section{Materials and Methods:}

Study Design:

This is a prospective study in which sample are those who presented with cervical lymphadenopathy and who have undergone FNAC and biopsy procedure along with other necessary investigations.

Place of Study:

In this study patients were randomly collected from inpatient and outpatient departments of Pediatric Medicine and Pediatric Surgery of Bangabandhu Sheikh Mujiib Medical University, Dhaka.

Duration of Study:

This study was carried out from July 2003 to June 2005 , a period of twenty four months.

Sampling Technique and Selection Criteria:

For patient selection, the following inclusion and exclusion criteria were followed.

Inclusion Criteria:

Patients of both sexes of pediatric age group (0-15 years) with enlarged cervical lymph nodes.

Exclusion Criteria:

1. Patients who had not undergone FNAC and biopsy examination.

2. Non specific lymphadenitis cases, where clinical features are obvious for diagnosis

3. Cases where available investigations revealed the diagnosis and cytology or histopathology of lymph nodes was not required e.g. Leukaemia.

Sample Size:

The study includes 50 cases of enlarged cervical lymph nodes.

Data Collection:

1. From preset questionnaire

2. Method of assessment:

- Clinical examination

- Relevant investigations 
Data Analysis:

All the data were analyses and calculated by standard statistical formulas with the help of resource personnel in the field of Biostatistics by using SPSS programmer.

\section{Ethical Consideration:}

1. Informed written consent from the guardians.

2. Relevant permission from ethical committee of BSMMU.

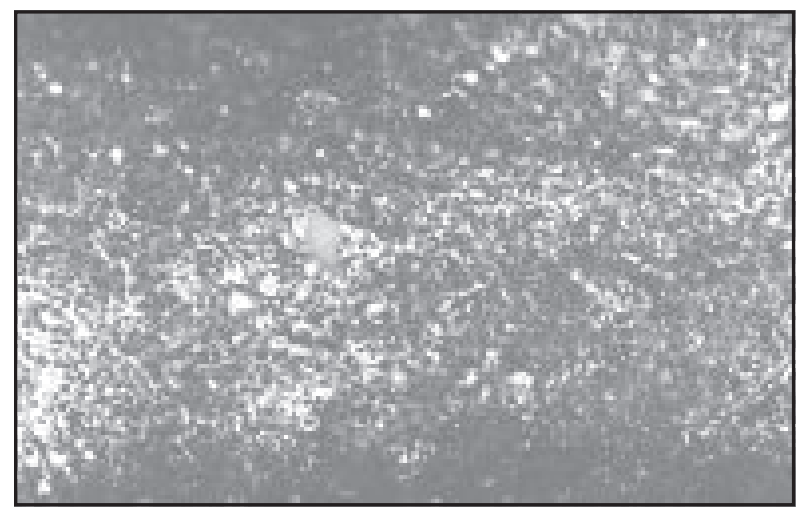

Reactive Node. Tissue section reveals follicular hyperplasia

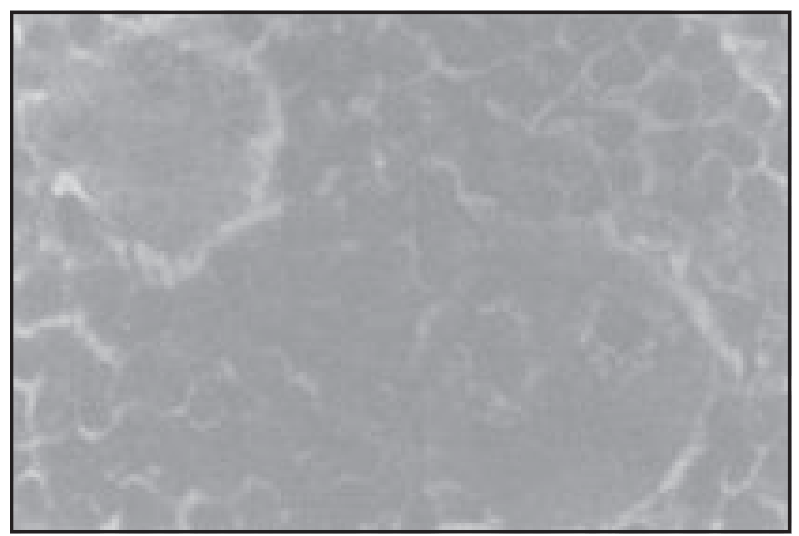

Hodgkin's Disease. Tissue Section Reveals Reed Sternberg Giant Cell

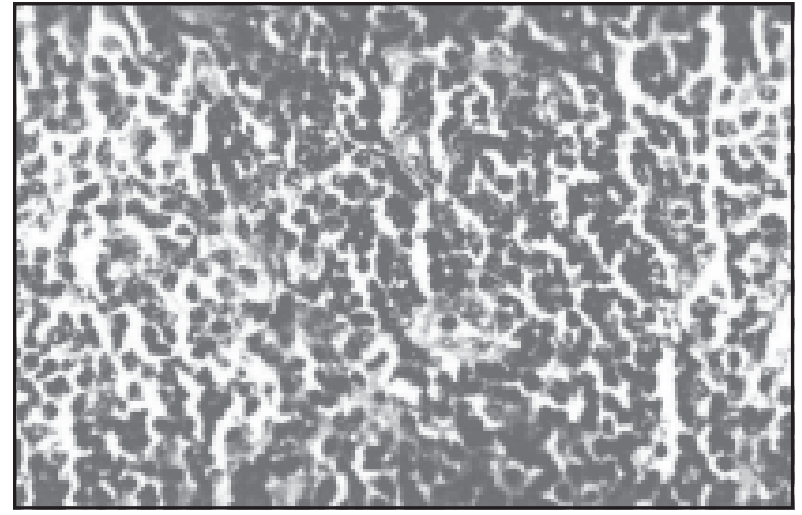

Non Hodgkin's Lymphoa. Tiussue Section Reveals Diffuse Small Cell Type Intermediate Grade

\section{Results:}

Out of total 50 cases, $31(62 \%)$ cases of enlarged cervical lymphnode were within a 10-15 years of age. Tuberculosis was the most prominent diagnosis in all age groups. Out of total 50 cases, $23(46 \%)$ were male and $27(54 \%)$ were female. The male to female ratio was $0.85: 1$. Most of the Lymphadenopathy had duration less than 6 months (46\%). Only $12 \%$ were of more than 6 months duration.

Among the total 50 cases, 30 cases were clinically positive for tuberculosis and 20 cases were negative for tuberculosis. On FNAC, 27 cases were truly positive (TP), 3 cases were false positive (FP), 3 cases were false negative (FN) and 17 cases were truly negative (TN) for tuberculosis. So, FNAC was positive for tuberculosis in 30 cases.

Among the total 50 cases, 15 cases were clinically diagnosed as lymphoma and 35 cases were clinically negative for lymphoma. On FNAC, 12 cases were true positive, 3 cases were false positive, 2 cases were false negative and 33 cases were true negative for lymphoma. So, a total of 14 cases were positive for lymphoma on FNAC.

Among the 50 cases, 5 cases were clinically diagnosed as nonspecific lymphadenitis and the rest 45 cases were clinically diagnosed either as tuberculous lymphadenitis or as lymphoma. On FNAC, 3 cases were true positive, 2 were false positive, 3 were false negative and 42 cases were true negative for nonspecific lymphadenitis. So, a total of 6 cases were FNAC positive for nonspecific lymphadenitis.

Diagnostic sensitivity, specificity and accuracy of FNAC in comparison to clinical findings in case of tuberculosis are $90 \%, 85 \%$ and $88 \%$ respectively.

Diagnostic sensitivity, specificity and accuracy of FNAC in comparison to clinical findings in case of lymphoma are $80 \%, 94.3 \%$ and $90 \%$ respectively.

Diagnostic sensitivity, specificity and accuracy of FNAC in comparison to clinical findings in case of reactive hyperplasia were $60 \%, 93.3$ and $90 \%$ respectively.

Among the 30 FNAC positive cases for tuberculosis, 25 cases were positive for tuberculosis on biopsy. Among the rest 20 FNAC negative cases for tuberculosis, 2 cases were confirmed as tuberculous lymphadenopathy on biopsy. So, a total of 27 cases were confirmed to be of tuberculosis.

Among the 14 FNAC positive cases for lymphoma, 13 were confirmed as lymphoma on biopsy. Among the rest FNAC negative cases of lymphoma, 2 cases 
were confirmed as lymphoma on biopsy. So, a total of 15 cases were confirmed to be due to lymphoma.

Among the 6 FNAC positive cases of nonspecific lymphadenitis, 4 cases were confirmed as such on biopsy. Among the rest 44 FNAC negative cases, 4 cases were confirmed as reactive hyperplasia on biopsy. So, a total of 8 cases were confirmed to have reactive hyperplasia.
Diagnostic sensitivity, specificity and accuracy of FNAC in comparison to histopathology for tuberculosis, are $92.5 \%, 78.3 \%$ and $86 \%$ respectively. In cases of lymphoma, they are $86.7 \%, 97.1 \%$ and $94 \%$ respectively.

Diagnostic sensitivity, specificity and accuracy of FNAC in comparison to histopathological findings for reactive hyperplasia are $50 \%, 95.3 \%$ and $83 \%$ respectively.

Table-I

Relation between FNAC and clinical findings

\begin{tabular}{lcccccc}
\hline Parameter & $\begin{array}{c}\text { Clinical } \\
\text { No. }\end{array}$ & findings & \multicolumn{2}{c}{ FNAC } & \multicolumn{2}{c}{$\begin{array}{c}\text { Findings } \\
\text { Positive }\end{array}$} \\
& & & & No. & $(\%)$ & \multicolumn{2}{c}{ No. } & $(\%)$ \\
\hline $\begin{array}{l}\text { Tuberculosis } \\
\text { Positive }\end{array}$ & 30 & $(60.0)$ & 27 & $(90.0)$ & 3 & $(10.0)$ \\
Negative & 20 & $(40.0)$ & 3 & $(15.0)$ & 17 & $(85.0)$ \\
Total: & 50 & & 30 & $(60.0)$ & 20 & $(40.0)$ \\
Lymphoma & & & & & & \\
Positive & 15 & $(30.0)$ & 12 & $(80.0)$ & 3 & $(20.0)$ \\
Negative & 35 & $(70.0)$ & 2 & $(5.7)$ & 33 & $(94.3)$ \\
Total: & 50 & & 14 & $(28.0)$ & 36 & $(72.0)$ \\
Nonspecific lymphadenitis & & & & & & \\
Positive & 5 & $(10.0)$ & 3 & $(60.0)$ & 2 & $(40.0)$ \\
Negative & 45 & $(90.0)$ & 3 & $(6.7)$ & 42 & $(95.5)$ \\
\hline
\end{tabular}

Table-II

Diagnostic accuracy of FNAC in comparison to clinical findings

\begin{tabular}{lccccc}
\hline Parameter & $\begin{array}{c}\text { Sensitivity } \\
(\%)\end{array}$ & $\begin{array}{c}\text { Specificity } \\
(\%)\end{array}$ & $\begin{array}{c}\text { Positive predictive } \\
\text { value }(\%)\end{array}$ & $\begin{array}{c}\text { Negative predictive } \\
\text { value }(\%)\end{array}$ & $\begin{array}{c}\text { Accuracy } \\
(\%)\end{array}$ \\
\hline Tuberculosis & 90.00 & 85.00 & 90.00 & 85.00 & 88.00 \\
Lymphoma & 80.00 & 94.29 & 85.71 & 91.67 & 90.00 \\
Nonspecific lymphadenitis & 60.00 & 93.33 & 50.00 & 95.45 & 90.00 \\
\hline
\end{tabular}

Table-III

Relation between FNAC and histopathology

\begin{tabular}{lcccccc}
\hline Parameter & $\begin{array}{c}\text { Histopathological findings } \\
\text { No. }\end{array}$ & $(\%)$ & \multicolumn{2}{c}{ FNAC } & \multicolumn{2}{c}{$\begin{array}{c}\text { Findings } \\
\text { Positive }\end{array}$} \\
& & & No. & $(\%)$ & No. & $\begin{array}{c}\text { Negative } \\
(\%)\end{array}$ \\
\hline Tuberculosis & 27 & $(54.0)$ & 25 & $(92.6)$ & 2 & $(7.4)$ \\
Positive & 23 & $(46.0)$ & 5 & $(21.7)$ & 18 & $(78.3)$ \\
Negative & 50 & & 30 & $(60.0)$ & 20 & $(40.0)$ \\
Total: & & & & & & \\
Lymphoma & 15 & $(30.0)$ & 13 & $(86.7)$ & 2 & $(13.3)$ \\
Positive & 35 & $(70.0)$ & 1 & $(2.9)$ & 34 & $(97.1)$ \\
Negative & 50 & & 14 & $(28.0)$ & 36 & $(72.0)$ \\
Total: & & & & & & \\
Nonspecific lymphadenitis & & 4 & $(50.0)$ & 4 & $(50.0)$ \\
Positive & 8 & $(16.0)$ & 2 & $(4.8)$ & 40 & $(95.2)$ \\
Negative & 42 & $(84.0)$ & 6 & $(12.0)$ & 44 & $(88.0)$ \\
\hline Total: & 50 & & & & & \\
\hline
\end{tabular}


Table 4

Diagnostic accuracy of FNAC in comparison to histopathological findings

\begin{tabular}{lccccc}
\hline Parameter & $\begin{array}{c}\text { Sensitivity } \\
(\%)\end{array}$ & $\begin{array}{c}\text { Specificity } \\
(\%)\end{array}$ & $\begin{array}{c}\text { Positive predictive } \\
\text { value }(\%)\end{array}$ & $\begin{array}{c}\text { Negative predictive } \\
\text { value }(\%)\end{array}$ & $\begin{array}{c}\text { Accuracy } \\
(\%)\end{array}$ \\
\hline Tuberculosis & 92.59 & 78.26 & 90.00 & 85.00 & 88.00 \\
Lymphoma & 86.67 & 97.14 & 92.86 & 94.44 & 94.00 \\
Nonspecific lymphadenitis & 50.00 & 95.24 & 66.67 & 90.91 & 88.00 \\
\hline
\end{tabular}

\section{Discussion:}

Cervical lymphadenopathy is quite a common condition throughout the world and more so in the developing countries like Bangladesh. There are several reasons why lymphadenopathy in the neck is more common than in other parts of the body. These are:

1. More than one third of the total body lymphnodes are distributed throughout the neck. ${ }^{1}$

2. Cervical lymph nodes are affected by various regional and systemic diseases. Cervical lymph nodes are the draining nodes of mouth and throat which are frequent sites of infection. ${ }^{8}$

Diagnosis of the exact cause of lymphadenopathy at the tissue level is mandatory before specific treatment can be given.

Excisional biopsy is still widely practiced for the diagnosis of lymphadenopathy in all central and many of the peripheral hospitals in the developing countries like Bangladesh.

Excisional biopsy is costly, time-consuming and intensive technique where sometimes hospitalization and even general anesthesia is required. ${ }^{1}$

In comparison to open surgical biopsy, FNAC is a simple reliable and acceptable tool for the etiological diagnosis of lymphadenopathies. ${ }^{9}$

It is very well-tolerated by children and no complication is encountered. ${ }^{10}$

Initially, 56 cases were randomly selected for the present study. Out of these 56 cases, 6 cases were deleted from the study, as smears were inadequate and hence only 50 cases were available for the study.

In all the 50 cases, included in the present study, FNAC and biopsy reports were available in all the cases and were compared with each other and with clinical findings. The role of FNAC was evaluated in various lesions of lymph nodes.
In the present study Tuberculosis is the commonest cause of cervical lymphadenopathy which comprises of $54 \%$ cases. Similar results were found by others. ${ }^{11}$

Some authors in Saudi Arabia, Korea and South Africa showed lower incidence of tuberculous lymphadenopathies. Lower incidence in these studies may be related to the high socio economic condition in these geographical areas. ${ }^{12}$

Over the last decade mycobacterium infections have been shown to be on rise worldwide and in the developing countries like Bangladesh, the incidence is very high.

The second most common cause of cervical lymphadenopathy in the present study is lymphoma, which comprises $30 \%$ of the cases. Out of which Hodgkin's Lymphoma is in $6(12 \%)$ and non-Hodgkin's Lymphoma is in $9(18 \%)$ cases. Similar results were observed by other authers. ${ }^{9,13}$

Cervical lymphadenopathy for non specific lymphadenitis (reactive change) is found in $16 \%$ cases in the present study. Acute cases were not included in the series as they were not subjected to FNAC or biopsy. Similar results are observed by others. ${ }^{9,12}$

In this study, of the total 50 cases $23(46 \%)$ cases are male and $27(54 \%)$ cases are female. The female to male ratio is $1: 0.88$. This data correlates with other reported series of cervical lymphadenopathies. ${ }^{14,15}$

The present study shows age range from 0-15 years. The highest number of cervical lymphadenopathy cases are seen in patients with 10-15 years of age. The similar age incidences were shown by others. ${ }^{12,15,16}$

Duration of lymphadenopathy is of less than 6 months in $88 \%$ cases and only in $12 \%$ cases duration of lymphadenopathy is of more than 6 months.

In the present study, 38 patients (76\%) belong to lower socio economic group and 12 patients (24\%) belong to middle class. 
In the present study, in a total of 50 cases, 30 cases are clinically diagnosed as tuberculous lymphadenitis, 15 cases as lymphoma and 5 cases as reactive hyperplasia due to nonspecific lymphadenitis. On FNAC, 30 cases are diagnosed as tuberculous lymphadenitis, 14 cases as lymphoma and 6 cases as nonspecific lymphadenitis. On biopsy, 27 cases are confirmed as tuberculosis, 15 cases as lymphoma, 8 cases as nonspecific lymphadenitis. Among the lymphoma cases, 6 are Hodgkin's Lymphoma and 9 are non-Hodgkin's Lymphoma.

Among the total 50 cases, 30 cases were clinically positive for tuberculosis and 20 cases were negative for tuberculosis. On FNAC, 27 cases were truly positive (TP), 3 cases were false positive (FP), 3 cases were false negative (FN) and 17 cases were truly negative (TN) for tuberculosis. So, FNAC was positive for tuberculosis in 30 cases.

Diagnostic sensitivity, specificity and accuracy of FNAC in comparison to clinical findings in case of tuberculosis are $90 \%, 85 \%$ and $88 \%$ respectively, in the present study.

Among the 30 FNAC positive cases for tuberculosis, 25 cases were positive for tuberculosis on biopsy. Among the rest 20 FNAC negative cases for tuberculosis, 2 cases were confirmed as tuberculous lymphadenopathy on biopsy. So, a total of 27 cases were confirmed to be of tuberculosis.

Diagnostic sensitivity, specificity and accuracy of FNAC in comparison to histopathology for tuberculosis, in the present study are $92.5 \% 78.3 \%$ and $86 \%$ respectively. These findings are consistent with those of the other studies. ${ }^{9,20}$

This study finds that FNAC is a very useful diagnostic tool to identify the patients of tuberculous lymphadenopathy positively. It obviates the need for excisional biopsy in most of the patients. Similar views were expressed by others. ${ }^{11,21}$

A thorough history, physical examinations, tuberculin test and fine needle aspiration will help to arrive at an early diagnosis of tubercular cervical lymphadenitis adenitis and allow early commencement of treatment before final diagnosis can be made by biopsy and culture. ${ }^{20}$

There are some problems in arriving at a definitive diagnosis in case of tuberculous lymphadenopathy particularly when Langhan's Giant cells and epitheloid cells are not seen in the smear. Similarly, the aspirate may contain only gaseous material or pus. ${ }^{9}$
False positive diagnosis of tuberculosis may arise due to polymorphic picture of smear without epitheloid cell, foamy macrophages or small clusters of histiocytes. Sometimes endothelial cells and dry artifact may look like epitheloid cells and diagnosis of tuberculosis can be made wrongly. The diagnostic error may be sampling error as a part of ineffective penetration of the tubercle by the needle. Investigators have observed that sometimes smears of tubercular patients may contain polymorphic picture without epitheloid cell or giant cell, making it impossible to differentiate from reactive change. It is, therefore, required to perform biopsy in such false negative cases to confirm the cytological diagnosis, where strong clinical suspicion of tuberculosis exists. For the same reason where positive cytodiagnosis does not correlate with clinical findings, biopsy should be done to substantiate FNAC diagnosis.

Among the total 50 cases, 15 cases were clinically diagnosed as lymphoma and 35 cases were clinically negative for lymphoma. On FNAC, 12 cases were true positive, 3 cases were false positive, 2 cases were false negative and 33 cases were true negative for lymphoma. So, a total of 14 cases were positive for lymphoma on FNAC.

Diagnostic sensitivity, specificity and accuracy of FNAC in comparison to clinical findings in case of lymphoma are $80 \%, 94.3 \%$ and $90 \%$ respectively.

Among the 14 FNAC positive cases for lymphoma, 13 were confirmed as lymphoma on biopsy. Among the rest FNAC negative cases of lymphoma, 2 cases were confirmed as lymphoma on biopsy. So, a total of 15 cases were confirmed to be due to lymphoma.

Diagnostic sensitivity, specificity and accuracy of FNAC in comparison to histopathological findings in cases of lymphoma are $86.7 \%, 97.1 \%$ and $94 \%$ respectively. This observation correlates with other studies. ${ }^{22,23}$

On aspiration cytology, Hodgkin's Lymphoma may show polymorphous picture as do tuberculous lymphadenitis and reactive hyperplasia. Therefore, ascertaining the presence of characteristic ReedSternberg cells in the smears is mandatory before making the diagnosis of Hodgkin's Lymphoma on aspiration cytology. On aspiration cytology, nonHodgkin's Lymphoma is characterized by a monomorphic appearance of the smear. Possible diagnostic error in case of non-Hodgkin's Lymphoma 
can arise from occasional admixture of other cells with monomorphic cells. ${ }^{9}$

It has also been claimed that, $22-36 \%$ cases of nonHodgkin's Lymphoma cannot be differentiated from nonspecific lymphadenitis due to minimum cytological atypia. ${ }^{24}$

Therefore, biopsy must be performed to confirm the cytological diagnosis of non-Hodgkin's Lymphoma.

Among the 50 cases, 5 cases were clinically diagnosed as nonspecific lymphadenitis and the rest 45 cases were clinically diagnosed either as tuberculous lymphadenitis or as lymphoma. On FNAC, 3 cases were true positive, 2 were false positive, 3 were false negative and 42 cases were true negative for nonspecific lymphadenitis. So, a total of 6 cases were FNAC positive for nonspecific lymphadenitis.

Diagnostic sensitivity, specificity and accuracy of FNAC in comparison to clinical findings were $60 \%$, $93.3 \%$ and $90 \%$ respectively.

Among the 6 FNAC positive cases of nonspecific lymphadenitis, 4 cases were confirmed as such on biopsy. Among the rest 44 FNAC negative cases, 4 cases were confirmed as reactive hyperplasia on biopsy. So, a total of 8 cases were confirmed to have reactive hyperplasia.

Diagnostic sensitivity, specificity and accuracy of FNAC in comparison to histopathological findings in cases of nonspecific lymphadenitis are 50\%, 95.3\% and $83 \%$ respectively. Similar results were observed by others. ${ }^{22,23,24}$ It is sometimes very difficult to comment on aspirates showing reactive hyperplasia. These aspirates show polymorphous picture with the presence of characteristic plasma cells. The possibility of a smear's reactive hyperplasia is enhanced by the presence of "tangible" bodies (macrophages with ingested debris) and mitotic figures. Patients with tuberculous lymphadenitis or Hodgkin's Lymphoma may have a similar polymorphous picture on aspirates. In the absence of characteristic epitheloid cells / Langhan's giant cells in the former and Reed-Sternberg cells in the later, it is almost impossible to differentiate such cases from reactive hyperplasia. ${ }^{1}$

Most of the cases of all non-diagnostic biopsies (i.e. reactive changes) eventually showed a disease related to the indication of biopsy. $91 \%$ of which did so within 6 months. $17-21 \%$ had a diagnosis of specific disease. Of the patients who subsequently were found to have lymphoma, $90 \%$ had their conditions diagnosed within 8 months of first biopsy. So, all cases of this group should be carefully followed up. ${ }^{25}$

It is difficult to determine the exact length of time for which an enlarged lymph node should be followed up in asymptomatic patients. If nodes have increased in size over the baseline measurements at follow up after 2 weeks, a biopsy is recommended. Even if it has no regress in size over 4 weeks, biopsy should be taken. ${ }^{26,27}$

The present study indicates that, FNAC is a simple, reliable and acceptable procedure for various lesions of cervical lymph nodes. It can be repeated if necessary. It also concludes that FNAC helps to confirm the clinical impression without open biopsy. Open biopsy can be avoided in many benign and malignant lesions of cervical lymph nodes.

Utilizing electron microscopy and immunohistochemical technique, along with flow cytometry, can greatly broaden the diagnostic range and specificity of fine needle aspiration cytology. ${ }^{28}$

Ultrasonography and CT scan can help in guiding FNAC for a more accurate cytological examination. ${ }^{29}$

Application of molecular techniques will provide conclusive pieces of information and consequently will enhance the efficiency of FNA in non-Hodgkin's Lymphoma. ${ }^{30}$

\section{Conclusion:}

FNAC is a very useful diagnostic tool in significant cases of lymphadenopathies. While tuberculous lymphadenopathy and Hodgkin's Lymphoma can be diagnosed by FNAC with a high degree of accuracy, the differentiating features are not well demarcated in reactive hyperplasic and non-Hodgkin's Lymphoma. Continued perseverance, patience and experience definitely can improve the results. FNAC will become a viable tool in the diagnosis of significant cases of lymphadenopathies in developing countries like Bangladesh.

However, the limitations of FNAC should be kept in mind and biopsy should be performed in any doubtful case.

As Stewart so rightly commented in 1993 and it is just as appropriate today: "Diagnosis by aspiration is as reliable as the combined intelligence of the clinician and pathologist makes it." 31 


\section{References:}

1. Cotran, R.S. Kumar, V. \& Collins,T. 1999, Robins Pathological Basis of Disease, 60' edn., W.B. Saunders Company, Philadelphia/ London/ Toronto/ Tokyo, pp.644-696.

2. Gupta, R.K. 1997, 'Fine Needle Aspiration of Toxoplasmic Lymphadenitis', Acta Cytologica, vol.41, no. 14, pp. 1031-4.

3. Junquira, L.C., Carneiro, J. \& Kelley, R.O. 1998, Basic Histopathology, 9th edn. A Lange Medical Publication, pp.259-262.

4. Al Fallouji, M.A.R. 1998, Post Graduate Surgery, The Candidates Guide, $2^{\text {nd }}$ edn, p.378

5. Mayer, S 'n.d.', 'Fine Needle Aspiration CytologyAn Old Technology Revised', J. of FNA Clinics of America.

6. Aretz, H.T. et al. 1984, 'Fine-needle Aspiration Why It Deserves Another Look', J. of Post Graduate Medicine, vol.75, no.3, pp.49-56.

7. Margolis et al. 1978, 'To Improve the Yeild of Biopsy of the Lyrnphnodes', Surgery, Gynaecology \& Obstetrics, vol.147, pp.376-378.

8. Russel, R.C, Williamas, N.S \& Bulstrode, C.J.K (eds) 2000, Baily and Love's Short Practice of Surgery, 23rd edn. Arnold International Students' Edition, London, pp.704-704.

9. Gupta, A ' K, Nayar, m \& Chandra, M 1991, 'Reliability \& Limitation of Fine Needle Aspiration Cytology of Lymphadenopathies, An Analysis of 1,261 Cases', Acta Cytologica, vol.35, no.6, pp.777-783.

10. Ramadan, H.H, Wax, MX \& Boyd, C.B 1997, 'Fine-needle Aspiration of Head and Neck masses in Children', Am. J Otolaryngology, vol.18, no.6, pp.400-4.

11. Andapat, M.C.D. et al. 1987, 'Diagnosis of Tuberculous Lymphadenitis by Fine-needle Aspiration Cytology', Ind J. Tab., vol.34, no. 139, pp. 12- 15 .

12. Al Sohaibani, M.O. 1996, 'Cervical Lymphadenopathy in Eastrn Province of Saudi Arabia', East Afr. Med J, vol.73, no.8, pp.533-7.

13. Haque, M.A. \& Talukder, S.I. 2003, 'Evaluation of Fine-needle Aspiration Cytology (FNAC) of Lymphnode in Mymensingh', Mymensingh Med.I., vol.21, no.l,i pp.33-5.
14. Das Gupta et al. 1994, 'Fine needle Aspiration Cytology of Cervical Lymphadenopathy with Special Reference to Tube4rculosis', JIMA, vol.92, no. 1, pp.

15. Jha, B.C. et al. 2001,'Cervical Lymphadenopathy: Changing Clinical Pattern and Concept in Management', Post Graduate Med. J, vol.77, pp. 185-187.

16. Parma, S.P \& Mathur, G.P 1974, 'A Cooperative Study of Tuberculous Cervical Lymphadenitis', Indian Med. J. Research, vol.62, no. 11, pp. 16311646.

17. Geldmacher et al. 2002, 'Assessment of Lymphnode Tuberculosis in Northern Germany', Chest Joumal,vol. 12 1, pp. 1177-1182.

18. Kheiry, J. \& Ahmed, M.E. 1992,'Cervical Lymphadenopathy in Khartoum', J Trop. Med. \& Hyg. vol.95, no.6, pp.416-419.

19. Kanlikama, M. et al. 2000,'Management Strategy of Mycobacterial Cervical Lymphadenopathy', J. of Laryngology \& Otology, vol. 114, pp.274-278.

20. Ibekwe, A.O., al Sharef, Z. \& al Kindy, S. 1997, 'Diagnostic Problem of Tuberculous Cervical Lymphadenitis (Scrofula), Am. J. Otolaryngol., vol. 18 , no.8, pp.202-5.

21. Pandit, A.A, Candes, F.P \& Khubchandani, S.R 1987, 'Fine Needle Aspirsation Cytology of Lymphnodes', Journal of Post Graduate Medicine, vol.33, no.3, pp. 134-6.

22. Pilotti,S et al. 1993, 'Diagnostic Assessment of Enlarged Lymphnodes by Fine Needle Aspiration', Acia Cytologica, vol.37, pp.853-66.

23. Al-Mulhim et al. 2004, 'The Role of Fine-needle Aspiration Cytology \& Imprint Cytology in Cervical Lymphadenopathy', Saudi Med J., vol. 25, no.7, pp.862-5.

24. Suen, C.K 1990, Atlas and Text of Aspiration, Biopsy, Cytology, Williams \& Wilkins Company, Baltimore, pp. 1 -3, 59-73.

25. Thomas, J.0, Adeyi, A.D \& Amanguno, H 1999, 'Fine Needle Aspiration in the Management of Peripheral; Lymphadnopathy in a Developing Country', Diagn. Cytopathology, vol.21. no.3, pp. 1596-162. 
26. Saltzstein, S.L 1965, 'The Fate of Patients with Nondiagnostic Lymphnode Biopsies', Surgery, vol.58, pp.659-662.

27. Slap, G.B et al. 1984, 'When to Perform Biopsies of Enlarged Peripheral Lympbnodes in Young Patients',.JIMA, vol.252, no.2, pp.] 324-1326.

28. Layfield, L.J. 1996,'Fine-needle Aspiration of Head and Neck', Pathology (Pilo), vol.4, no.2, pp.403-38.

29. Ying, M, Ahuja A.T \& Yuen, H.Y 2004, ‘Greyscale and power Doppler Sonography of Unusual
Cervical Lymphadenopathy', Ultrasound Med. Biology, vol.30, no.4, pp.449-54.

30. Manitchotpisit, B et al. 1991, 'Combined use of Fine-needle AspirationCytology and Polymerase Chain Reaction in the Diagnosis of Cervical Tuberculous Lymphadenitis', J. Med. Assoc. Thailand, vol.82, no.4, pp.363-8.

31. Lew, W.Y.C. 1987, 'Fine Needle Aspiration Cytology: A Personal Experience with 800 Cases', Singapore Med. J. pp.214-219. 